\title{
Preoperative Prognostic Value of Dynamic Contrast-Enhanced MRI-Derived Contrast Transfer Coefficient and Plasma Volume in Patients with Cerebral Gliomas
}

T.B. Nguyen, G.O. Cron, J.F. Mercier, C. Foottit, C.H. Torres, S. Chakraborty, J. Woulfe, G.H. Jansen, J.M. Caudrelier, J. Sinclair, M.J. Hogan, R.E. Thornhill, and I.G. Cameron

\begin{abstract}
BACKGROUND AND PURPOSE: The prognostic value of dynamic contrast-enhanced MR imaging-derived plasma volume obtained in tumor and the contrast transfer coefficient has not been well-established in patients with gliomas. We determined whether plasma volume and contrast transfer coefficient in tumor correlated with survival in patients with gliomas in addition to other factors such as age, type of surgery, preoperative Karnofsky score, contrast enhancement, and histopathologic grade.
\end{abstract}

MATERIALS AND METHODS: This prospective study included 46 patients with a new pathologically confirmed diagnosis of glioma. The contrast transfer coefficient and plasma volume obtained in tumor maps were calculated directly from the signal-intensity curve without $\mathrm{T} 1$ measurements, and values were obtained from multiple small ROls placed within tumors. Survival curve analysis was performed by dichotomizing patients into groups of high and low contrast transfer coefficient and plasma volume. Univariate analysis was performed by using dynamic contrast-enhanced parameters and clinical factors. Factors that were significant on univariate analysis were entered into multivariate analysis.

RESULTS: For all patients with gliomas, survival was worse for groups of patients with high contrast transfer coefficient and plasma volume obtained in tumor $(P<.05)$. In subgroups of high- and low-grade gliomas, survival was worse for groups of patients with high contrast transfer coefficient and plasma volume obtained in tumor $(P<.05)$. Univariate analysis showed that factors associated with lower survival were age older than 50 years, low Karnofsky score, biopsy-only versus resection, marked contrast enhancement versus no/mild enhancement, high contrast transfer coefficient, and high plasma volume obtained in tumor $(P<.05)$. In multivariate analysis, a low Karnofsky score, biopsy versus resection in combination with marked contrast enhancement, and a high contrast transfer coefficient were associated with lower survival rates $(P<.05)$.

CONCLUSIONS: In patients with glioma, those with a high contrast transfer coefficient have lower survival than those with low parameters.

ABBREVIATIONS: $\mathrm{DCE}=$ dynamic contrast-enhanced; $\mathrm{HGG}=$ high-grade glioma; $\mathrm{HR}=$ hazard ratio; $K^{\mathrm{trans}}=$ contrast transfer coefficient; $\mathrm{rCBV}=$ relative cerebral blood volume; $\mathrm{V}_{\mathrm{p}}=$ plasma volume

n patients presenting with cerebral gliomas, the World Health Organization tumor grade is an important prognostic factor along with clinical and radiologic findings such as age, preoperative Karnofsky performance status, and the presence of contrast enhancement. ${ }^{1-4}$ The extent of tumor resection is another known

Received December 12, 2013; accepted after revision March 10, 2014.

From the Departments of Diagnostic Imaging (T.B.N., G.O.C., C.H.T., R.E.T., I.G.C., S.C., J.M.C.), Medical Physics (C.F., I.G.C.), Pathology (G.H.J., J.W.), Surgery, Division of Neurosurgery (J.S.), and Medicine, Division of Neurology (M.J.H.), The Ottawa Hospital, University of Ottawa, Ottawa, Ontario, Canada; and Department of Radiology (J.F.M.), Hôpital de Hull, Gatineau, Québec, Canada.

This work was supported by the Brain Tumour Foundation of Canada.

Please address correspondence to Thanh Binh Nguyen, MD, Department of Diagnostic Imaging, The Ottawa Hospital, 1053 Carling Ave, Ottawa, Ontario, K1Y 4E9

Canada; e-mail: thnguyen@toh.on.ca

http://dx.doi.org/10.3174/ajnr.A4006 factor affecting patient survival. ${ }^{1}$ Recently, by using dynamic susceptibility contrast MR imaging, the relative CBV was identified as an important prognostic marker for survival independent of tumor grade. ${ }^{5-7}$ For example, Law et $\mathrm{al}^{5}$ have shown that gliomas with high relative cerebral blood volume $(\mathrm{rCBV})(>1.75)$ have a shorter time to progression compared with tumors with similar grades but lower rCBV, regardless of tumor grade.

While dynamic susceptibility contrast-derived rCBV has been shown to predict survival in patients with gliomas in single-center studies, the need for normalization of semiquantitative values with a manually defined reference region can introduce interpatient and interscanner variability and thus limits its use in a multiinstitutional clinical trial. ${ }^{8}$ Furthermore, measurements can be biased by $\mathrm{T} 1$ effects due to extravascular contrast leakage in tumor vessels. ${ }^{9,10}$ Hemodynamic parameters are more readily quantified 
by using a dynamic contrast-enhanced (DCE) MR imaging technique, which allows simultaneous measurement of CBV and the contrast transfer coefficient $\left(K^{\text {trans }}\right) . K^{\text {trans }}$ is the volume transfer coefficient of contrast from the vascular compartment to the extravascular compartment. It is influenced by cerebral blood flow and vascular permeability. ${ }^{11}$ While $K^{\text {trans }}$ has been found to increase with glioma grade, its prognostic value remains unclear. ${ }^{12,13}$ A previous study reported that in patients with highgrade gliomas (HGGs), higher values of $K^{\text {trans }}$ were associated with longer survival. ${ }^{12}$ This finding is unexpected because high $K^{\text {trans }}$ implies, in theory, a higher level of angiogenesis, greater biologic aggressiveness, and a shorter survival.

The goal of our study was to determine the prognostic values of $K^{\text {trans }}$ and plasma volume $\left(\mathrm{V}_{\mathrm{p}}\right)$ obtained in tumor by using a simple DCE MR imaging acquisition that relies on the change in signal intensity rather than the $\mathrm{T} 1$ relaxation rate.

\section{MATERIALS AND METHODS Subjects}

All examinations were conducted in accordance with the guidelines for human research at our institution, and written informed consent was obtained from all participating subjects. All adult patients presenting at The Ottawa Hospital with a newly diagnosed brain lesion compatible with a glioma between December 2008 and March 2011 were included in this prospective study. We set the following exclusion criteria: prior surgery or biopsy of the presenting brain lesion, pregnancy, renal failure, and a known history of allergy to gadolinium-based MR imaging contrast agent.

We recorded clinical variables such as the following: age, sex, use of steroids before preoperative MR imaging, and the preoperative Karnofsky score. Following MR imaging, patients underwent surgery (biopsy or resection), depending on tumor size, tumor location, and the patient's general condition. The median time interval between the MR imaging examination and surgery was 5.0 days (95\% CI, 3.9-7.0 days). Histopathologic diagnosis was provided by an experienced neuropathologist (J.W., with 12 years of experience, or G.H.J., with 23 years of experience) by using the World Health Organization classification. From the operative report, the type of surgery was classified as biopsy or resection. The main outcome measure was overall survival. Follow-up clinical information was obtained primarily from chart review, telephone interview, and archived obituaries. The follow-up period was defined as the interval between the date of surgery and the date of death or the date the patient was last known to be alive. Follow-up was halted on December 12, 2012.

\section{MR Imaging Acquisition}

All preoperative and postoperative MR imaging data were acquired by using either a 1.5T (Symphony; Siemens, Erlangen, Germany) or a 3T clinical scanner (Magnetom Trio; Siemens). Preoperative anatomic imaging was performed by using standard sagittal T1-weighted, axial T1-weighted pre- and postcontrast injection, axial FLAIR, axial T2, and coronal T1 postcontrast injection sequences.

DCE MR imaging was performed following the axial T1weighted precontrast imaging. At 1.5T, DCE MR imaging was performed by using a 2D FLASH pulse sequence ( 5 axial sections centered on the tumor, $\mathrm{TR}=45 \mathrm{~ms}, \mathrm{TE}=2.1,5.5 \mathrm{~ms}$, flip angle $=$ $90^{\circ}$, matrix $=96 \times 128, \mathrm{FOV}=17 \times 23 \mathrm{~cm}^{2}$, section thickness $=$ $5 \mathrm{~mm}, \Delta \mathrm{t}=2.2$ seconds). 2D FLASH was used for dynamic imaging on the 1.5T scanner due to hardware limitations because the desired temporal resolution ( $\Delta \mathrm{t}<3$ seconds) could only be achieved with $2 \mathrm{D}$ sequences. At $3 \mathrm{~T}$, a 3D FLASH sequence was used ( 18 axial sections, $\mathrm{TR}=6.5 \mathrm{~ms}, \mathrm{TE}=1.7,3.9 \mathrm{~ms}$, flip angle $=$ $30^{\circ}$, matrix $=96 \times 128, \mathrm{FOV}=23 \mathrm{~cm}^{2}$, section thickness $=5$ $\mathrm{mm}, \Delta \mathrm{t}=2.9$ seconds).

Gadopentetate dimeglumine (Magnevist; Bayer Pharma, Berlin, Germany) was injected at $0.1 \mathrm{mmol} / \mathrm{kg}$ and $4 \mathrm{~mL} / \mathrm{s}$, beginning 40 seconds after the start of the scan. The duration of the DCE sequence was 220 seconds.

\section{Pharmacokinetic Modeling}

DCE magnitude images were processed directly in nordicICE, Version 2 (NordicNeuroLab, Bergen, Norway) to generate maps of $\mathrm{V}_{\mathrm{p}}$ and $K^{\text {trans }}$ with the assumption that changes in contrast concentration are proportional to changes in MR imaging signal intensity with the same proportionality constant in tissue and in blood. Kinetic parameters $\left(K^{\text {trans }}\right.$ and $\mathrm{V}_{\mathrm{p}}$ ) were estimated by using a 2-compartment extended Tofts model implemented in the nordicICE software. ${ }^{14}$ The vascular input function was calculated from the superior sagittal sinus. ${ }^{15}$

\section{Image Interpretation}

A neuroradiologist (C.H.T.) blinded to the histopathologic grade determined the degree of contrast enhancement relative to the choroid plexus: none, mild (less than that of the choroid plexus), or marked (equal to or more than that of the choroid plexus).

A senior radiology resident (J.F.M, third-year radiology resident) traced 4 ROIs (each $25 \mathrm{~mm}^{2}$ ) in the solid part of the tumor in the areas of visually highest $\mathrm{V}_{\mathrm{p}} / K^{\text {trans }}$ present on the acquired sections. The maximum value among the 4 region-of-interest values was obtained for $K^{\text {trans }}$ and $V_{p}$ for each patient. All ROIs were verified by a neuroradiologist (T.B.N., with 10 years of experience) to ensure that inadvertent placement on an adjacent vessel was avoided.

\section{Statistical Analysis}

All data were analyzed by using MedCalc for Windows, Version 11.5 (MedCalc Software, Mariakerke, Belgium). Patients were divided into 2 groups based on their tumor values of $\mathrm{V}_{\mathrm{p}}$ and $K^{\text {trans }}$. Threshold values of 0.05 minutes $^{-1}$ and $5 \%$ for $K^{\text {trans }}$ and $V_{p}$, respectively, were chosen for simplicity and convenience so that they might be used in clinical practice and for comparison with other related studies. We checked that each group had at least 30\% of patients. Kaplan-Meier survival curves were obtained for patients with no or mild enhancement versus marked enhancement and for high tumor $\mathrm{V}_{p} / K^{\text {trans }}$ versus low $\mathrm{V}_{\mathrm{p}} / K^{\text {trans }}$. Kaplan-Meier survival analysis was also performed for the subgroups of patients with low- and high-grade gliomas. Potential prognostic variables for time-to-death were explored by using Cox proportional hazards modeling. We selected clinical/radiologic/histopathologic variables such as tumor grade, age older than 50 years, Karnofsky performance status score lower than 80 , tumor size of $>4 \mathrm{~cm}$, 
Table 1: Summary of clinical characteristics as univariate predictors of overall survival in 46 patients with gliomas

\begin{tabular}{|c|c|c|c|c|}
\hline Characteristics & No. & $\%$ & $\mathrm{HR}(95 \% \mathrm{Cl})$ & $P$ Value \\
\hline \multicolumn{5}{|l|}{ Age } \\
\hline 50 Years or younger & 18 & 39.1 & 1 & \\
\hline Older than 50 years & 28 & 60.9 & $7.62(2.17-26.71)$ & .002 \\
\hline \multicolumn{5}{|l|}{ Karnofsky performance score } \\
\hline$\geq 80$ & 25 & 54.3 & 1 & \\
\hline$<80$ & 21 & 45.7 & $2.86(1.35-6.08)$ & .007 \\
\hline \multicolumn{5}{|l|}{ Maximal tumor diameter } \\
\hline$<4 \mathrm{~cm}$ & 17 & 37 & 1 & \\
\hline$\geq 4 \mathrm{~cm}$ & 29 & 63 & $1.30(0.61-2.80)$ & .50 \\
\hline \multicolumn{5}{|l|}{ Surgery } \\
\hline Biopsy only & 9 & 19.6 & 1 & \\
\hline Resection & 37 & 80.4 & $4.19(1.77-9.93)$ & .001 \\
\hline \multicolumn{5}{|l|}{ Histopathologic grade } \\
\hline Grade 2 (8 pure astrocytomas, 1 oligodendroglioma) & 9 & 19.6 & 1 & \\
\hline $\begin{array}{l}\text { Grade } 3 \text { (6 pure astrocytomas, } 2 \text { oligoastrocytomas, } \\
1 \text { oligodendroglioma) }\end{array}$ & 9 & 19.6 & $0.087(0.83-9.45)$ & .87 \\
\hline Grade 4 (26 pure astrocytomas, 2 oligoastrocytomas) & 28 & 40.8 & $4.14(1.25-13.8)$ & .02 \\
\hline \multicolumn{5}{|c|}{ Steroid administration } \\
\hline No & 18 & 39.1 & 1 & \\
\hline Yes & 28 & 61.9 & $2.11(0.96-4.65)$ & .06 \\
\hline
\end{tabular}

Table 2: Summary of imaging characteristics as univariate predictors of overall survival in 46 patients with gliomas

\begin{tabular}{lcccc}
\multicolumn{1}{c}{ Characteristics } & No. & $\%$ & HR (95\% Cl) & $P$ Value \\
\hline Sequence & & & & \\
$\quad$ 2D & 31 & 67 & 1 & \\
3D & 15 & 33 & $1.00(0.44$ to 2.29) & .99 \\
$\begin{array}{l}\text { Contrast enhancement } \\
\quad \text { None/less than that of choroid plexus }\end{array}$ & 15 & 33 & 1 & \\
$\quad$ Equal/more than that of choroid plexus & 31 & 67 & $8.11(2.44-26.90)$ & .0007 \\
$K^{\text {trans }\left(\text { min }^{-1}\right)}$ & & & & \\
$\quad<0.05$ & 17 & 37.0 & 1 & \\
$\geq 0.05$ & 29 & 63.0 & $7.63(2.63-22.11)$ & .0002 \\
$V_{\mathrm{p}}(\mathrm{mL} / 100 \mathrm{~g})$ & & & & \\
$\quad<5$ & 20 & 43.5 & 1 & .003 \\
$\geq 5$ & 26 & 56.5 & $4.17(1.77-9.80)$ & .001 \\
\hline
\end{tabular}

radiation and chemotherapy. Among 37 patients with HGGs, 1 had an oligodendroglioma, 4 had oligoastrocytomas, and 32 had pure astrocytomas. Thirty-four patients underwent standard chemotherapy and radiation treatment. One patient was enrolled in a clinical trial combining temozolomide with bevacizumab or a placebo. Twenty-eight patients (4 with low-grade gliomas and 24 with HGGs) received steroids before their MR imaging at admission compared with 18 who did not (5 low-grade gliomas and 13 HGGs). In patients with HGGs, no statistically significant difference was found in the median $V_{p}$ or $K^{\text {trans }}$ between patients who received steroids versus those who did not $(P>.64)$.

\section{Kaplan-Meier Survival Curve Analysis}

Twenty-nine deaths occurred. The median follow-up for those still alive was 836 days. The median survival time for all patients was 597 days with a 1-year survival probability of .62 .

\section{Histopathologic Grade}

Patients with glioblastoma multiforme had a statistically significant worse survival rate compared with those with grades 3 and $2(P<.05$, Fig $1 A)$. There was no statistical difference found in the survival curves for patients with grade 3 versus 2 . When we compared those with grade 2 gliomas who had a diagnosis from

biopsy versus resection, and degree of tumor enhancement because these variables have been reported as prognostic factors. ${ }^{1-4}$ Clinical and imaging variables that resulted in a probability $<.05$ on univariate analysis were entered into multivariate analysis in a forward stepwise regression. Multivariate analysis was performed for 3 models, each model including an imaging variable and clinical variables. For each model, a variable is entered into the model with $P<.05$. Significant variables are entered sequentially; after a variable is entered in the model, variables that became nonsignificant $(P>.1)$ are removed. Degree of contrast enhancement, $K^{\text {trans }}$, and $\mathrm{V}_{\mathrm{p}}$ were not entered in the same model because they are not independent variables.

\section{RESULTS}

\section{Study Population}

Forty-six patients with gliomas were included in this study. Seven patients were recruited but were excluded due to lack of a surgical biopsy $(n=2)$ or the presence of metastatic disease on histopathologic examination $(n=5)$. Patient demographics, tumor size, and histopathologic grade are summarized in Table 1, while DCE MR imaging parameters are summarized in Table 2. Among 9 patients with a low-grade glioma, 1 had an oligodendroglioma and 8 had pure astrocytomas. Four of those patients underwent a surgical resection (not from a biopsy) with those with grade 3 gliomas, a trend of longer survival for low-grade gliomas was seen, though it was not statistically significant $(P=.2$, Fig $1 B)$.

\section{Degree of Contrast Enhancement}

There was a statistical difference in survival between groups with no or mild enhancement versus marked enhancement for all patients $(P=.0001)$ and for subgroups of low-grade gliomas $(P=$ $.002)$ and HGGs $(P=.01)$. For patients with anaplastic astrocytomas, marked enhancement was associated with worse survival $(P=.04)$. Among patients with glioblastoma multiforme, 1 had mild enhancement and 1 did not have any enhancement. Their survival rate was not different from those with marked enhancement $(P=.81)$.

\section{$K^{\text {trans }}$ and $\boldsymbol{V}_{\boldsymbol{p}}$}

For all gliomas, a statistically significant difference was present in the comparison of survival curves between groups with low and high $K^{\text {trans }}$ and $\mathrm{V}_{\mathrm{p}}(P<.05$, Figs 2 and 3$)$. Subgroup analysis of patients with HGGs and low-grade gliomas also revealed significant decreases in survival rates in the high $K^{\text {trans }}$ and $\mathrm{V}_{\mathrm{p}}$ groups compared with their respective "low" counterparts. Further sub- 
group analysis of patients with HGGs who underwent resection showed significant decreases in survival rates for patients with high $K^{\text {trans }}(P=.007)$, but not for patients with high $\mathrm{V}_{\mathrm{p}}(P=.08)$.

Table 3: Three models combining an imaging parameter with clinical factors as potential prognostic variables of overall survival in patients with gliomas

\begin{tabular}{|c|c|c|}
\hline Factors & $\mathrm{HR}(95 \% \mathrm{Cl})$ & $P$ Value \\
\hline \multicolumn{3}{|l|}{ Model 1} \\
\hline Age older than $50 \mathrm{yr}$ & $1.03(0.99-1.06)$ & .17 \\
\hline Biopsy vs resection & $4.11(1.68-10.05)$ & .002 \\
\hline Grade 3 vs 2 & $1.20(0.19-7.65)$ & .85 \\
\hline Grade 4 vs 2 & $1.21(0.28-5.33)$ & .80 \\
\hline Karnofsky score $<80$ & $2.56(1.19-5.51)$ & .02 \\
\hline$K^{\text {trans }} \geq 0.05 \mathrm{~min}^{-1}$ & $4.53(1.22-16.82)$ & .02 \\
\hline \multicolumn{3}{|l|}{ Model 2} \\
\hline Age older than $50 \mathrm{yr}$ & $1.06(1.03-1.09)$ & .0002 \\
\hline Biopsy vs resection & $3.68(1.54-8.78)$ & .004 \\
\hline Grade 3 vs 2 & $1.06(0.16-6.89)$ & .95 \\
\hline Grade 4 vs 2 & $1.76(0.35-8.75)$ & .49 \\
\hline Karnofsky score $<80$ & $3.21(1.48-6.92)$ & .003 \\
\hline $\mathrm{V}_{\mathrm{p}} \geq 5 \mathrm{~mL} / 100 \mathrm{~g}$ & $1.30(0.37-4.54)$ & .68 \\
\hline \multicolumn{3}{|l|}{ Model 3} \\
\hline Age older than $50 \mathrm{yr}$ & $1.03(0.99-1.07)$ & .087 \\
\hline Biopsy vs resection & $4.36(1.77-10.77)$ & .002 \\
\hline Grade 3 vs 2 & $1.28(0.20-8.25)$ & .79 \\
\hline Grade 4 vs 2 & $1.00(0.22-4.63)$ & .99 \\
\hline Karnofsky score $<80$ & $2.51(1.16-5.43)$ & .02 \\
\hline Marked contrast enhancement & $4.79(1.22-18.87)$ & .03 \\
\hline
\end{tabular}

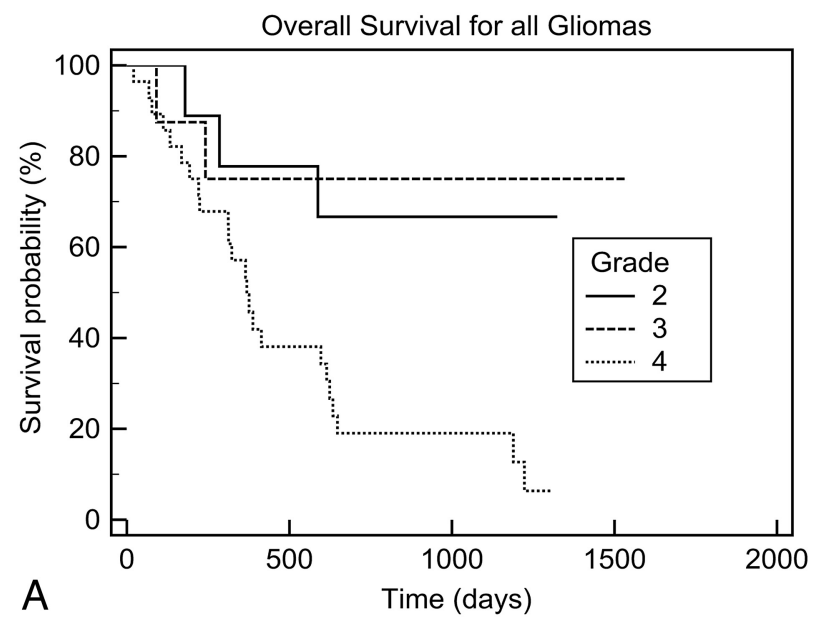

In patients with anaplastic astrocytomas, those with high $K^{\text {trans }}$ and high $\mathrm{V}_{\mathrm{p}}$ had a worse prognosis $(P=.04)$. In patients with glioblastoma multiforme, there was a trend toward shorter survival for patients with high $K^{\text {trans }} /$ high $V_{p}$, but this did not reach statistical significance $(P=.26$ and $P=.50$, respectively).

\section{$2 D$ versus $3 D$ Sequence}

We did not find any significant differences in survival rates between groups who had 2D versus $3 \mathrm{D}$ sequences for all gliomas and for subgroups of low-grade gliomas and HGGs $(P>.5)$.

\section{Univariate Predictors of Overall Survival}

Univariate predictors of poorer survival were age older than 50 years, preoperative Karnofsky score of $<80$, biopsy only, grade 4 , marked contrast enhancement, and high $K^{\text {trans }}$ and $\mathrm{V}_{\mathrm{p}}$ values (Table 1). We did not find tumor size a significant prognostic variable $(P=.5)$. Patients who were prescribed steroids at admission before their MR imaging examination appear to have a poorer prognosis (hazard ratio $[\mathrm{HR}]=2.11,95 \% \mathrm{CI}$ $0.96-4.65)$ than those who were not, but there were more HGGs among them. We found a statistically significant difference in survival between grades 4 and $3(\mathrm{HR}=4.77,95 \% \mathrm{CI}$, 1.13-20) and grades 4 and $2(\mathrm{HR}=4.14,95 \% \mathrm{CI}, 1.25-13.8)$, but not between grades 3 and 2 ( $\mathrm{HR}=0.087,95 \% \mathrm{CI}, 0.83-$ 9.45). When we compared the prognostic value of the $2 \mathrm{DCE}$

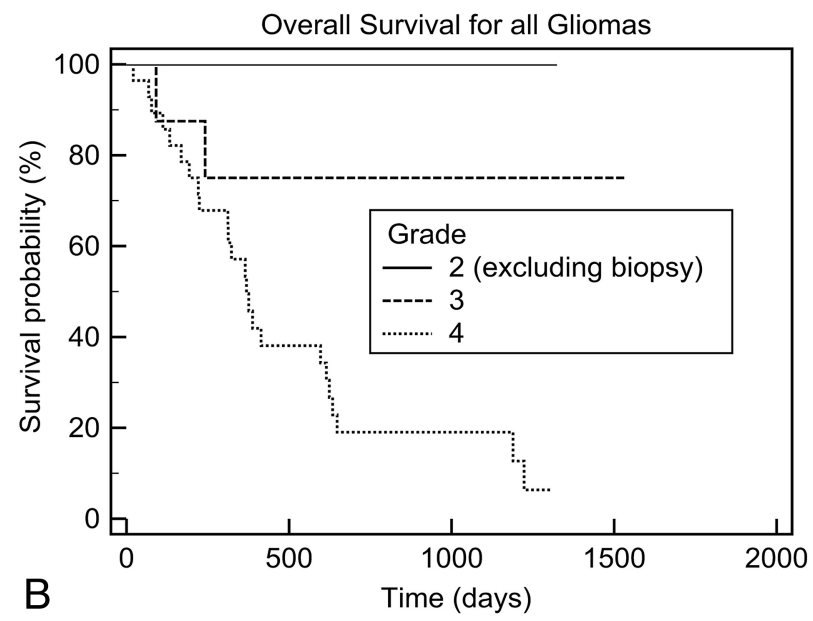

FIG 1. Kaplan-Meier analysis of overall survival according to histopathologic grades. A, All patients (between grades 2 and $3, P=.8$; and between grades 3 and $4, P=.02$ ). $B$, All patients, excluding those with low-grade gliomas who had a biopsy (between grades 2 and $3, P=.2$; between grades 3 and $4, P=.02$ ).
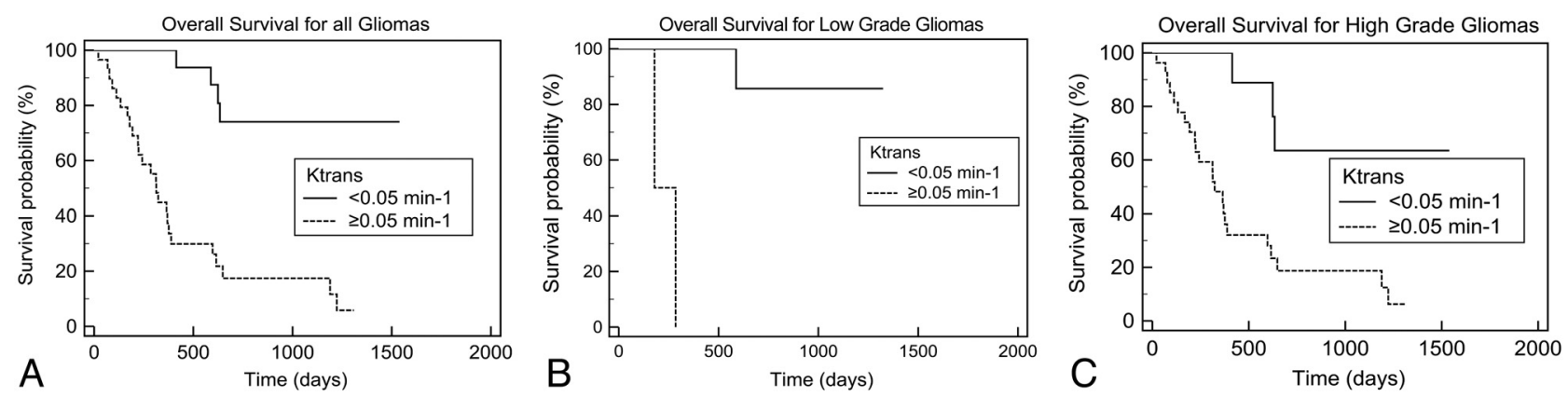

FIG 2. Kaplan-Meier analysis of overall survival according to $K^{\text {trans }}$. A, All patients $(P=.0001)$. $B$, Those with low-grade gliomas $(P=.002)$. $C$, Those with high-grade gliomas $(P=.004)$. 

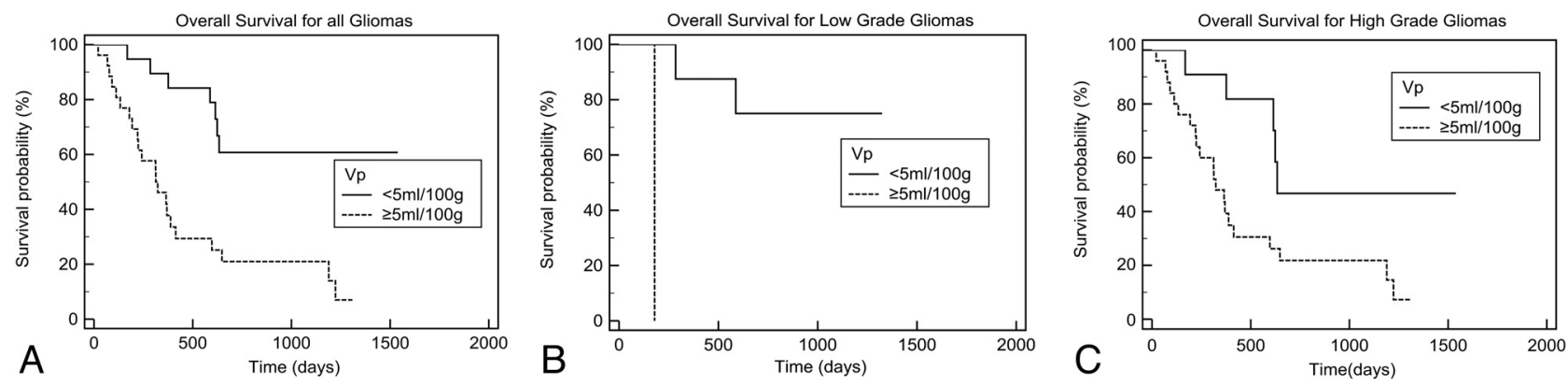

FIG 3. Kaplan-Meier analysis of overall survival according to $V_{p}$. $A$, All patients $(P=.0004)$. $B$, Those with low-grade gliomas $(P=.005)$. $C$, Those with high-grade gliomas $(P=.02)$.

parameters, patients with high $K^{\text {trans }}$ had a higher hazard of mortality $(\mathrm{HR}=7.63,95 \% \mathrm{CI}, 2.63-22.11)$ compared with $\mathrm{V}_{\mathrm{p}}$ $(\mathrm{HR}=4.17,95 \% \mathrm{CI}, 1.77-9.80)$. Patients with marked tumor enhancement also had a higher hazard of mortality $(\mathrm{HR}=$ 8.11, 95\% CI, 2.44-26.90).

\section{Multivariate Predictors of Overall Survival}

In multivariate analysis combining clinical and imaging parameters, Karnofsky score of $<80$ and biopsy were the only significant clinically poor prognostic factors in any model (Table 3 ). Age older than 50 years was a significant prognostic factor in only 1 of the 3 models. Histopathologic grading and $V_{p}$ did not remain significant factors in our multivariate analysis. High $K^{\text {trans }}$ and contrast enhancement were significant poor prognostic factors, along with biopsy and Karnofsky score of $<80$.

\section{DISCUSSION}

In our study of patients with gliomas, preoperative Karnofsky performance score and the type of surgery were important prognostic factors in both univariate and multivariate analysis, in agreement with the findings in previous studies. ${ }^{1-3}$ Grade IV was found to be a poor prognostic factor compared with grades 3 and 2 in univariate analysis. However, we did not observe a significant difference in survival for grade 3 versus 2 . Because some of our patients with grade 2 were diagnosed via biopsy only, sampling error could have led to an improper grading of the tumor, which could explain why we did not observe a statistical difference in survival rates between patients with grade 2 and those with grade 3 gliomas.

With dynamic susceptibility contrast MR imaging, numerous previous articles have recently established $\mathrm{rCBV}$ as a prognostic factor in patients with low- and high-grade gliomas. ${ }^{5-7}$ However, use of DSC-derived rCBV as a potential biomarker in a multiinstitutional trial is currently limited because it is a semiquantitative measurement, which can be influenced by many postprocessing steps, including the technique of correction for contrast agent extravasation and the choice of the normal contralateral white matter. ${ }^{8,9}$ For patients with gliomas, CBV obtained from the normal contralateral white matter has been shown to have significant interscanner variability. ${ }^{8}$ For DCE, measurement of absolute CBV might be possible because correction of contrast leakage across tumor vessels can be more accurately quantified by using a proper pharmacokinetic model. Furthermore, DCE is not influenced by susceptibility artifacts, which can make measurements of rCBV by using DSC difficult in gliomas with hemorrhagic components or gliomas located near the skull base.

In patients with gliomas, the use of DCE imaging as a potential biomarker for prognosis has not been well studied. We have found that marked contrast tumoral enhancement and high $V_{p}$ were negative prognostic factors in all patients with gliomas and in the subgroups of patients with high- and low-grade gliomas. While contrast enhancement remains a significant factor in multivariate analysis, $\mathrm{V}_{\mathrm{p}}$ and histopathologic grade did not add any prognostic value when age, type of surgery, and Karnofsky performance status score were also included. Because the hazard ratios for $\mathrm{V}_{\mathrm{p}}$ and grade were less than those for age and type of surgery in univariate analysis, we believe that the small sample size in our study did not allow detection of those weaker prognostic factors in multivariate analysis.

High $K^{\text {trans }}$ had the same negative prognostic value as marked contrast tumoral enhancement in patients with gliomas and in subgroups of patients with high- and low-grade gliomas. While measurement of $K^{\text {trans }}$ requires some postprocessing, $K^{\text {trans }}$ might represent a more objective metric in the setting of a multicenter clinical trial because qualitative assessment of the degree of contrast enhancement is subject to interobserver variability. The prognostic value of $K^{\text {trans }}$ is similar to that in our findings with CT perfusion. ${ }^{16}$ In a different cohort of 20 patients with high-grade gliomas who underwent preoperative CT perfusion, we found that those with a combination of high CBV and permeability surface had an HR of 6.0 (95\% CI, 4.2-7.8,) compared with those with lower values. Mathematic modeling based on DCE MR imaging revealed that $K^{\text {trans }}$ was correlated with tumor aggressiveness, perhaps due to the increased vessel growth and permeability required for cellular proliferation. ${ }^{13}$ However, Mills et $\mathrm{al}^{12}$ observed a positive correlation between $K^{\text {trans }}$ and prognosis in their group of 19 patients with high-grade gliomas, which led to their hypothesis that tumors with higher baseline $K^{\text {trans }}$ might be more responsive to radiation treatment or chemotherapy than those with lower $K^{\text {trans }}$. Another study by Awasthi et $\mathrm{al}^{17}$ found that high $K^{\text {trans }} /$ volume of extravascular space was correlated with a high expression of matrix metalloproteinases and was associated with poorer survival.

These conflicting results on the prognostic value of $K^{\text {trans }}$ could be due to the small sample size, different effects of covariate factors (such as the extent of tumor resection, use of radiation treatment or chemotherapy, and molecular/genomic markers), 
and/or different DCE MR imaging methods. ${ }^{18,19}$ The method for DCE MR imaging-parameter estimation can influence the magnitude of the hazard ratio. Our method did not use baseline T1 measurements to convert signal intensity to gadolinium concentration as suggested by DCE MR guidelines published by Leach et al. ${ }^{20}$ This simpler method can yield more useful measurements when the signal intensity-to-noise ratio is low, such as in poorly enhancing tumors. $^{21}$ The method is implemented on commercially available software, and postprocessing time is relatively short.

Our study was limited by the small number of patients, especially those with low-grade gliomas and nonenhancing tumors. A second limitation is that the location of the tumor and the treatment received after surgery were not included as independent variables in our study. For example, 3 patients with glioblastomas did not undergo radiation treatment. This could have explained why we did not find tumor size a statistically significant prognostic factor. We are also aware of potential measurement bias due to methodologic differences between the 2D and 3D techniques used in our study. However, we do not believe that the different pulse sequence and field strengths had a major systematic effect on the pharmacokinetic parameters in our study. Although, in theory, 2D and $3 \mathrm{D}$ gradient-echo sequences might have different sensitivities to water exchange and inflow effects, we did not find a statistical difference in the mean and median values of pharmacokinetic parameters in groups of patients scanned with $2 \mathrm{D}$-versus-3D sequences. There was no significant difference in survival for patients with gliomas scanned with 2D-versus-3D sequences. MR imaging signal-intensity curves from the superior sagittal sinus and tumor were not systematically different between patients in the 2 groups.

Because many patients with gliomas received steroids at the time of admission, this treatment might have introduced a measurement bias on the pharmacokinetic parameters. However, in our study, we did not observe a statistical difference in $K^{\text {trans }}$ or $\mathrm{V}_{\mathrm{p}}$ in patients with grade 3 and 4 gliomas who were given steroids versus those who were not. Corticosteroid treatment has been reported to decrease contrast enhancement in malignant gliomas and to reduce total fractional blood volume. ${ }^{22,23}$ Animal studies have suggested that its antiangiogenic effects are both transient and vessel-size-dependent. In rats with gliomas treated with dexamethasone, rCBV was only significantly reduced on the third day following treatment. ${ }^{23}$ While rCBV measured by gradient-echo MR imaging was reduced $>50 \%$, an increase in $\mathrm{rCBV}$ was observed with spin-echo MR imaging. ${ }^{24}$ Our study patients usually had started steroid treatment for only a short duration (usually $<72$ hours) before their MR imaging examination. This duration might explain why we did not see any significant effect on our hemodynamic parameters. This finding is in agreement with that in Bastin et $\mathrm{al},{ }^{25}$ who did not find a significant change in tumor CBV and CBF before and after $48-72$ hours of dexamethasone treatment.

\section{CONCLUSIONS}

Preoperative high $K^{\text {trans }}$ obtained from DCE MR imaging is associated with poorer outcome in patients with low- and high-grade gliomas. High tumoral $K^{\text {trans }}$ has the same prognostic value as the presence of marked tumoral enhancement on postcontrast T1weighted images.

\section{ACKNOWLEDGMENTS}

We thank William Petrcich for his expert statistical advice.

Disclosures: Thanh Binh Nguyen-RELATED: Grant: funding from the Brain Tumour Foundation of Canada,* UNRELATED: Board Membership, Consultancy, Grants/ Grants Pending, and Payment for the Development of Educational Presentations: all from Bayer Healthcare. Santanu Chakraborty-UNRELATED: Payment for lectures (including service on Speakers Bureaus): EMD Serono. Matthew J. HoganRELATED: Grant: Ottawa Hospital Research Institute and his laboratory have received in-kind support from GE Healthcare of approximately $\$ 11,000$ as part of an infrastructure grant from the Canadian Foundation for Innovation in 2011. *Money paid to the institution.

\section{REFERENCES}

1. Kalkanis SN, Rosenblum ML. Malignant gliomas. In: Bernstein M, Berger MS, eds. Neuro-oncology: The Essentials. 2nd ed. New York: Thieme Medical Publishers; 2008:254-65

2. Chaichana K, McGirt M, Niranjan A, et al. Prognostic significance of contrast-enhancing low-grade gliomas in adults and a review of the literature. Neurol Res 2009;31:931-39

3. Chang EF, Smith JS, Chang SM, et al. Preoperative prognostic classification system for hemispheric low-grade gliomas in adults. J Neurosurg 2008;109:817-24

4. Pope WB, Sayre J, Perlina A, et al. MR imaging correlates of survival in patients with high-grade gliomas. AJNR Am J Neuroradiol 26:2466-74

5. Law M, Young RJ, Babb JS, et al. Gliomas: predicting time to progression or survival with cerebral blood volume measurements at dynamic susceptibility-weighted contrast-enhanced perfusion MR imaging. Radiology 2008;247:490-98

6. Hipp SJ, Steffen-Smith E, Hammoud D, et al. Predicting outcome of children with diffuse intrinsic pontine gliomas using multiparametric imaging. Neuro Oncol 2011;13:904-09

7. Hirai T, Murakami R, Nakamura H, et al. Prognostic value of perfusion MR imaging of high-grade astrocytomas: long-term follow-up study. AJNR Am J Neuroradiol 2008;29:1505-10

8. Ellingson B, Zaw T, Cloughesy TF, et al. Comparison between intensity normalization techniques for dynamic susceptibility contrast (DSC)-MRI estimates of cerebral blood volume (CBV) in human gliomas. J Magn Reson Imaging 2012;25:1472-77

9. Boxerman JL, Schmainda KM, Weisskoff RM. Relative cerebral blood volume maps corrected for contrast agent extravasation significantly correlate with glioma tumor grade, whereas uncorrected maps do not. AJNR Am J Neuroradiol 2006;27:859-67

10. Boxerman JL, Prah DE, Paulson ES, et al. The role of preload and leakage correction in gadolinium-based cerebral blood volume estimation determined by comparison with MION as a criterion standard. AJNR Am J Neuroradiol 2012;33:1081-87

11. Henderson E, Sykes J, Drost D, et al. Simultaneous MRI measurement of blood flow, blood volume, and capillary permeability in mammary tumors using two different contrast agents. JMagn Reson Imaging 2000;12:991-1003

12. Mills SJ, Patankar TA, Haroon HA, et al. Do cerebral blood volume and contrast transfer coefficient predict prognosis in human glioma? AJNR Am J Neuroradiol 2006;27:853-58

13. Venkatasubramanian R, Arenas RB, Henson MA, et al. Mechanistic modelling of dynamic MRI data predicts that tumour heterogeneity decreases therapeutic response. Br J Cancer 2010;103:486-97

14. Tofts PS. Modeling tracer kinetics in dynamic Gd-DTPA MR imaging. J Magn Reson Imaging 1997;7:91-101

15. Nguyen TB, Cron GO, Mercier JF, et al. Diagnostic accuracy of dynamic contrast-enhanced MR imaging using a phase-derived vascular input function in the preoperative grading of gliomas. AJNR Am J Neuroradiol 2012;33:1539-45

16. Shankar JJ, Nguyen TB, Woulfe J, et al. Evaluation of CT perfusion in grading and prognostication of high grade gliomas: a pilot study. AJR Am J Roentgenol 2013;200:W504-09

17. Awasthi R, Pandey CM, Sahoo P, et al. Dynamic contrast-enhanced 
magnetic resonance imaging-derived $\mathrm{k}_{\mathrm{ep}}$ as potential biomarker of matrix metalloproteinase 9 expression in patients with glioblastoma multiforme: a pilot study. J Comput Assist Tomogr 2012; 36:125-30

18. Jain R, Poisson L, Narang J, et al. Genomic mapping and survival prediction in glioblastoma: molecular subclassification strengthened by hemodynamic imaging biomarkers. Radiology 2013;267: $212-20$

19. Takahashi $Y$, Nakamura H, Makino K, et al. Prognostic value of isocitrate dehydrogenase $1, \mathrm{O}^{6}$-methyguanine-DNA methyltransferase promoter methylation, and $1 \mathrm{p} 19 \mathrm{q}$ co-deletion in Japanese malignant glioma patients. World J Surg Oncol 2013;11:284

20. Leach MO, Brindle KM, Evelhoch JL, et al. The assessment of antiangiogenic and antivascular therapies in early-stage clinical trials using magnetic resonance imaging: issues and recommendations. Br J Cancer 2005;92:1599-610

21. Song HK, Xue Y, Yu J, et al. Comparison of the standard gadolin- ium concentration and signal difference methodologies for computation of perfusion parameters in DCE-MRI at various SNRs. In: Proceedings of the Annual Meeting of the International Society of Magnetic Resonance in Medicine, Montreal, Quebec, Canada. April 20-26, 2011

22. Zaki HS, Jenkinson MD, Duplessis DG, et al. Vanishing contrast enhancement in malignant glioma after corticosteroid treatment. Acta Neurochir (Wien) 2004;146:841-45

23. Darpolor M, Molthen RC, Schmainda KM. Multimodality imaging of abnormal vascular perfusion and morphology in preclinical 9L gliosarcoma model. PLoS One 2011;6:e16621

24. Badruddoja MA, Krouwer HG, Rand SD, et al. Antiangiogenic effects of dexamethasone in 9L gliosarcoma assessed by MRI cerebral blood volume maps. Neuro Oncol 2003;5:235-43

25. Bastin ME, Carpenter TK, Armitage PA, et al. Effects of dexamethasone on cerebral perfusion and water diffusion in patients with high-grade glioma. AJNR Am J Neuroradiol 2006;27:402-08 